\title{
Retention in care, resource utilization, and costs for adults receiving antiretroviral therapy in Zambia: a retrospective cohort study
}

\author{
Callie A Scott ${ }^{1,2}$, Hari S Iyer ${ }^{2}$, Kelly McCoy ${ }^{1}$, Crispin Moyo ${ }^{3,4}$, Lawrence Long ${ }^{5}$, Bruce A Larson 1,6*
} and Sydney Rosen ${ }^{1,5}$

\begin{abstract}
Background: Of the estimated 800,000 adults living with HIV in Zambia in 2011, roughly half were receiving antiretroviral therapy (ART). As treatment scale up continues, information on the care provided to patients after initiating ART can help guide decision-making. We estimated retention in care, the quantity of resources utilized, and costs for a retrospective cohort of adults initiating ART under routine clinical conditions in Zambia.

Methods: Data on resource utilization (antiretroviral [ARV] and non-ARV drugs, laboratory tests, outpatient clinic visits, and fixed resources) and retention in care were extracted from medical records for 846 patients who initiated ART at $\geq 15$ years of age at six treatment sites between July 2007 and October 2008. Unit costs were estimated from the provider's perspective using site- and country-level data and are reported in 2011 USD.

Results: Patients initiated ART at a median CD4 cell count of 145 cells/ $\mu$ L. Fifty-nine percent of patients initiated on a tenofovir-containing regimen, ranging from $15 \%$ to $86 \%$ depending on site. One year after ART initiation, $75 \%$ of patients were retained in care. The average cost per patient retained in care one year after ART initiation was \$243 (95\% Cl, \$194-\$293), ranging from \$184 (95\% Cl, \$172-\$195) to \$304 (95\% Cl, \$290-\$319) depending on site. Patients retained in care one year after ART initiation received, on average, 11.4 months' worth of ARV drugs, 1.5 CD4 tests, 1.3 blood chemistry tests, 1.4 full blood count tests, and 6.5 clinic visits with a doctor or clinical officer. At all sites, ARV drugs were the largest cost component, ranging from $38 \%$ to $84 \%$ of total costs, depending on site.

Conclusions: Patients initiate ART late in the course of disease progression and a large proportion drop out of care after initiation. The quantity of resources utilized and costs vary widely by site, and patients utilize a different mix of resources under routine clinical conditions than if they were receiving fully guideline-concordant care. Improving retention in care and guideline concordance, including increasing the use of tenofovir in first-line ART regimens, may lead to increases in overall treatment costs.
\end{abstract}

Keywords: HIV/AIDS, Adult, Antiretroviral therapy, Resource-limited setting, Costs, Outcomes, Zambia

\footnotetext{
* Correspondence: blarson@bu.edu

${ }^{1}$ Center for Global Health and Development, Boston University, Crosstown

Center, 3rd Floor, 801 Massachusetts Avenue, Boston, MA 02118, USA

${ }^{6}$ Department of International Health, School of Public Health, Boston

University, Crosstown Center, 3rd Floor, 801 Massachusetts Avenue, Boston,

MA 02118, USA

Full list of author information is available at the end of the article
} 


\section{Background}

Of the estimated 800,000 adults living with HIV in Zambia in 2011, roughly half were receiving antiretroviral therapy (ART) [1,2]. The Government of Zambia revised national HIV treatment guidelines for adults in 2007 to recommend the use of tenofovir as a standard component of first-line ART [3]. Guidelines were revised again in 2010 to raise the CD4 cell count threshold for ART eligibility from 200 cells $/ \mu \mathrm{L}$ to 350 cells $/ \mu \mathrm{L}$ and again in 2013 to remove any CD4 cell count threshold for ART eligibility for pregnant women [4,5]. Each of these changes substantially increased either the number of patients eligible for treatment or the cost of the drugs that comprise the treatment.

As treatment scale up continues, and as the Government of Zambia considers further guidelines changes that could expand ART eligibility even more or affect the quality of care for patients already on ART, information about the actual care provided to patients after initiating ART in Zambia can help guide decision making. Published papers have reported on the positive clinical and programmatic outcomes for patients initiating ART in Zambia [6,7] and on the costs of providing ART in Zambia [8,9], but no published papers have described both the actual care provided by the public sector clinics and hospitals that serve the vast majority of patients and the associated costs at the patient level. Our objective was to estimate retention in care, the quantity of resources utilized, and costs for adults initiating treatment under routine clinical conditions in Zambia.

\section{Methods}

\section{Analytic overview}

We enrolled a retrospective cohort of HIV-infected adults who initiated ART at six treatment sites in Zambia between July 2007 and October 2008, after tenofovir replaced stavudine in national guidelines as a standard component of first-line antiretroviral therapy. We collected patient-level data on retention in care and resource utilization from outpatient medical records. We estimated site- and country-level data on unit costs from financial reports, procurement records, and other sources. We estimated the proportion of patients retained in care, the average quantity of resources utilized per patient and per patient retained in care, and the average cost per patient and per patient retained in care through one year after ART initiation at all six sites and through two and three years after ART initiation at two of the six sites (where additional data were available at the time of data collection). We included resources utilized and costs incurred at the treatment site only; off-site resource utilization and costs were excluded. Costs were calculated from the provider's perspective in 2011 US dollars.

\section{Study sites}

Large scale, public sector provision of ART in Zambia began in Lusaka in 2004 and rapidly expanded. At the time of this study, clinics and hospitals across the country were providing ART, laboratory tests, and medications for opportunistic infections to patients free of charge. We purposively selected six of these sites to illustrate different models or settings for adult ART delivery in Zambia (Table 1). Sites included two primary health clinics in Lusaka Province (sites 1 and 2), a primary health clinic in Copperbelt Province (site 3), a second-level general hospital in Western Province (site 4), a first-level district hospital in Southern Province (site 5), and a second-level mission hospital in Southern Province (site 6). The number of active patients enrolled in the ART program at each site in 2008 ranged from 524 at site 5 to 5,748 at site 4 .

At the time of this study, ART eligibility, recommended ART regimens, and schedules for laboratory and clinical monitoring were in accordance with the 2007 national HIV treatment guidelines [3]. When CD4 testing was available, patients were eligible to initiate ART if they had (1) a CD4 $<200$ cells $/ \mu \mathrm{L},(2)$ a $C D 4<$ 350 cells $/ \mu \mathrm{L}$ with a WHO clinical stage 3 disease, or (3) a WHO clinical stage 4 disease. When CD4 testing was not available, patients were eligible to initiate ART if they had (1) a WHO clinical stage 3 or 4 disease or (2) a WHO clinical stage 2 disease and a total lymphocyte count $<1200$ cells $/ \mu \mathrm{L}$. Guidelines recommended a first-

Table 1 Study site characteristics

\begin{tabular}{|c|c|c|c|c|c|c|}
\hline Characteristic & Site 1 & Site 2 & Site 3 & Site 4 & Site 5 & Site 6 \\
\hline Province & Lusaka & Lusaka & Copperbelt & Western & Southern & Southern \\
\hline Facility type & $\begin{array}{l}\text { Primary } \\
\text { health clinic }\end{array}$ & $\begin{array}{l}\text { Primary } \\
\text { health clinic }\end{array}$ & $\begin{array}{l}\text { Primary health } \\
\text { clinic }\end{array}$ & $\begin{array}{l}\text { Second-level general } \\
\text { hospital }^{\mathrm{a}}\end{array}$ & $\begin{array}{l}\text { First-level district } \\
\text { hospital }^{a}\end{array}$ & $\begin{array}{l}\text { Second-level mission } \\
\text { hospital }^{a}\end{array}$ \\
\hline Setting & Urban & Urban & Urban & Urban & Urban & Rural \\
\hline $\begin{array}{l}\text { Number of active patients enrolled } \\
\text { in ART program, } 2008\end{array}$ & 5,488 & 5,102 & 1,167 & 5,748 & 524 & 2,981 \\
\hline
\end{tabular}


line ART regimen containing tenofovir, emtricitabine, and either efavirenz or nevirapine, with abacavir and lamivudine substituted for tenofovir and emtricitabine for patients with low creatinine clearance. Guidelines also recommended at least two CD4, hemoglobin, white blood cell count, and blood chemistry tests and at least five clinic visits during the first year on ART.

\section{Sample enrollment}

Adults who initiated ART at least 12 months prior to data collection at sites 1, 3, 4, and 5, and at least 36 months prior to data collection at sites 2 and 6, were selected consecutively from clinic registers and enrolled in the study. Data collection at sites 2 and 6 occurred after data collection at the other sites. Patients at sites 2 and 6 therefore had a longer follow-up period than patients at the other sites because additional data were available at the time of data collection.

Patients were eligible for enrollment if they were at least 15 years of age at the time of ART initiation and were not known to have transferred to another clinic during the study follow up period. The target enrollment at each site was 150 patients. Fewer patients were enrolled at site 5 due to low patient volume. Seven patients, one each at sites $1,2,4$, and 5 , and three at site 3 , were excluded after enrollment because they were found not to meet all study inclusion criteria. Total enrollment was therefore 846 patients.

\section{Ethics statement}

The Boston University Medical Center Institutional Review Board and the University of Zambia Biomedical Research Ethics Committee provided ethical approval of the study (protocol numbers H-28282 and 003-06-07). A waiver of informed consent was granted by both committees because the study was a retrospective review of routinely collected information from patient medical records.

\section{Data collection}

Data on patient outcomes and resources utilized at the treatment site during the first 12 or 36 months following ART initiation were obtained from each study patient's medical record. All resources used by the provider to deliver outpatient care to study patients were included, even if the resource cost was borne by another entity. Data on the costs of resources utilized were collected from site-level financial records, interviews with site managers, and country-level drug price lists and procurement records.

\section{Classification of patient outcomes}

Study patients at all sites were assigned outcomes at 12 months after initiating ART. Patients at sites 2 and 6 were also assigned outcomes at 24 and 36 months after initiating ART. Patients were classified as known to have died if a confirmation of death was noted in their medical record before the 12-, 24-, or 36-month endpoint. Patients were classified as lost to follow up if they were $\geq 3$ months late for their last scheduled consultation or medication pickup before the endpoint but had no confirmation of death in their medical record. Patients not classified as known to have died or lost to follow up were classified as retained in care.

\section{Estimation of unit costs}

We used previously published methods to estimate unit costs for fixed and variable resources utilized at the treatment sites by study patients, as outlined in Table 2 [10-13]. Fixed resources included buildings, equipment, and support staff employed in the ART clinic who do not see patients. Variable resources included ARV drugs, non-ARV drugs, laboratory tests, and provider time for clinic visits.

Costs estimated in Zambian Kwacha (ZMK) were first adjusted to 2011 levels, if necessary, using the consumer price index and then converted to US dollars at a rate of $4,861 \mathrm{ZMK} / \$$, the average exchange rate for 2011 $[14,15]$. We excluded costs of resources used for inpatient care and resources used for outpatient care above the level of the treatment site, such as government or NGO costs of oversight or training. We also excluded costs of resources procured by individual patients, such as transport to the clinic, and costs of resources used prior to ART initiation.

\section{Average resource utilization and costs}

We calculated the total quantity of each resource utilized by each patient and then calculated total costs for each patient by multiplying the unit cost for each resource by the total quantity utilized. We calculated average resource utilization and costs by dividing total resource utilization and costs for all patients by the total number of patients in the cohort. We repeated the same calculations for the subset of patients retained in care 12 months after initiating ART. At sites 2 and 6, where we had 36 months of follow up for each patient, we also calculated the average annual cost for the subset of patients retained in care 24 and 36 months after initiating ART by estimating the average cost for these patients over the first two or three years on ART and then dividing by two or three years.

We calculated average resource utilization and costs for each of sites 1 through 6 alone, as well as for sites 1 through 6 combined. The confidence intervals for the means for the six sites combined were adjusted for clustering at the site level (using the cluster option with the regression command in Stata). 
Table 2 Methods for estimating unit costs

\begin{tabular}{|c|c|}
\hline Resource & Method \\
\hline \multicolumn{2}{|c|}{ Fixed resources } \\
\hline $\begin{array}{l}\text { Buildings and } \\
\text { equipment }\end{array}$ & $\begin{array}{l}\text { Estimated upfront investment costs using a replacement cost approach, then calculated annualized costs using a } 3 \% \text { annual } \\
\text { discount rate and an estimated working life of } 5 \text { years for equipment and } 50 \text { years for buildings [11]. Annual building and } \\
\text { equipment costs for the ART clinic at each site were divided by the total number of active HIV patients at the site per year to } \\
\text { estimate a cost per patient-year in care. The total number of active HIV patients at the site was calculated by summing the } \\
\text { number of ART patients at the site with the number of non-ART patient-equivalents weighted based on a ratio of the average } \\
\text { number of patient visits to the site per year for non-ART versus ART patients. }\end{array}$ \\
\hline Support staff & $\begin{array}{l}\text { Estimated annual cost of support staff employed in the ART clinic at the study sites during the study period based on } 2011 \\
\text { salaries and allowances. The proportion of staff time allocated to ART versus non-ART activities was based on staff estimates. } \\
\text { Annual support staff costs were divided by the total number of active HIV patients at the site per year to estimate a cost } \\
\text { per patient-year in care. The total number of active HIV patients at the site was calculated by summing the number of ART } \\
\text { patients at the site with the number of non-ART patient-equivalents weighted based on a ratio of the average number of } \\
\text { patient visits to the site per year for non-ART versus ART patients. Costs for higher-level administrative support staff, including } \\
\text { staff based in the health facility in which the ART clinic was located, were excluded from the analysis. }\end{array}$ \\
\hline
\end{tabular}

Variable resources

ARV drugs Estimated as the average per unit cost for all units of a particular drug purchased for the Zambia national HIV program in 2011, or during the most recent year available if no units of a particular drug were purchased in 2011, as recorded in the Global Price Reporting Mechanism [12]. Data on ART regimen dispensed were used to determine the appropriate drug formulation (fixed dose tablet, single dose tablet) for each ARV drug dispensed [3].

Non-ARV drugs Estimated from standard Zambian Ministry of Health per package costs [13].

Laboratory tests

For sites 3, 4, 5, and 6, where laboratory tests are run onsite, costs were estimated as the sum of unit costs for reagents, consumables, equipment, labor, and space. Costs of reagents and consumables were estimated from standard Zambian Ministry of Health per package costs [13]. Annual laboratory equipment, labor, and space costs were divided by the total number of laboratory tests performed per year to estimate a per test cost at each site. For sites 1 and 2, where laboratory tests are run at a centralized laboratory, costs were provided by the Centre for Infectious Disease Research in Zambia Central Laboratory in Lusaka and included the cost of reagents, consumables, equipment, and labor used for each test (Henry Latner, personal communication, April 29, 2011).

Provider time for Estimated the total annual cost of staff time for each type of provider conducting patient consultations. The proportion of staff time allocated to ART versus non-ART activities was based on staff estimates. Cost per visit was calculated by dividing the total cost of staff time for each type of provider, valued at 2011 salaries and allowances, by the total number of patient consultations with each provider type per calendar year.

\section{Results}

\section{Cohort characteristics}

Patients initiated ART at a median age of 35 years and a median CD4 cell count of 145 cells $/ \mu \mathrm{L} ; 60 \%$ of patients were female (Table 3). The majority of patients (59\%) initiated on regimens containing tenofovir while the remainder initiated on zidovudine (14\%), abacavir (14\%), and stavudine (13\%). ART regimen at initiation varied widely by site.

\section{Patient outcomes}

One year after ART initiation, $75 \%$ of patients were retained in care, $11 \%$ were known to have died, and $15 \%$ were lost to follow up (Table 4). The proportion of

Table 3 Cohort characteristics

\begin{tabular}{|c|c|c|c|c|c|c|c|}
\hline Characteristic & Site 1 & Site 2 & Site 3 & Site 4 & Site 5 & Site 6 & All sites \\
\hline Total sample size & 149 & 149 & 147 & 149 & 102 & 150 & 846 \\
\hline Follow up time, months & 12 & 36 & 12 & 12 & 12 & 36 & - \\
\hline Median age at ART initiation, years [IQR] & $35[30-40]$ & $35[30-43]$ & $34[29-40]$ & $33[29-42]$ & $35[31-41]$ & $37[31-46]$ & $35[30-42]$ \\
\hline Sex, \% female & 63 & 52 & 68 & 66 & 45 & 58 & 60 \\
\hline Median CD4 at ART initiation, cells/ $\mu \mathrm{L}[\mathrm{IQR}]^{\mathrm{a}}$ & $149[97-211]$ & $127[67-206]$ & $136[84-199]$ & $150[77-237]$ & $140[74-273]$ & $160[106-209]$ & $145[86-212]$ \\
\hline \multicolumn{8}{|l|}{ Regimen at ART initiation, \% of patients } \\
\hline TDF-containing regimen & 36 & 86 & 80 & 15 & 54 & 80 & 59 \\
\hline AZT-containing regimen & 30 & 5 & 13 & 4 & 30 & 7 & 14 \\
\hline $\mathrm{ABC}$-containing regimen & 0 & 5 & 0 & 76 & 0 & 0 & 14 \\
\hline d4T-containing regimen & 34 & 5 & 7 & 5 & 16 & 13 & 13 \\
\hline
\end{tabular}

ART: antiretroviral therapy; ABC: abacavir; AZT: zidovudine; d4T: stavudine; IQR: interquartile range; TDF: tenofovir.

${ }^{\mathrm{a}} 793$ of 846 patients in the sample had a CD4 cell count at initiation. 
Table 4 Retention in care one year after initiating antiretroviral therapy

\begin{tabular}{llllllll}
\hline Patient outcome, $\mathbf{n}(\%)$ & Site $\mathbf{1}$ & Site $\mathbf{2}$ & Site $\mathbf{3}$ & Site $\mathbf{4}$ & Site $\mathbf{5}$ & Site $\mathbf{6}$ & All sites \\
\hline Retained in care & $116(78)$ & $103(69)$ & $109(74)$ & $116(78)$ & $76(75)$ & $113(75)$ & $633(75)$ \\
Known to have died & $13(9)$ & $23(15)$ & $14(10)$ & $7(5)$ & $6(6)$ & $26(17)$ & $89(11)$ \\
Lost to follow up & $20(13)$ & $23(15)$ & $24(16)$ & $26(17)$ & $20(20)$ & $11(7)$ & $124(15)$ \\
\hline
\end{tabular}

patients retained in care ranged from $69 \%$ to $78 \%$ depending on site.

At sites 2 and 6 , where patients were followed for three years after ART initiation, retention in care decreased from $69 \%$ and $75 \%$ one year after initiating ART to $64 \%$ and $72 \%$ two years after initiating ART and $61 \%$ and $64 \%$ three years after initiating ART. For patients retained in care one year after initiating ART at sites 2 and 6 , the probability of being retained in care two years after initiating ART was 93\%. For patients retained in care two years after initiating ART, the probability of being retained in care three years after initiating ART was $92 \%$. The proportion of patients known to have died was $18 \%$ two and three years after initiating ART.

\section{Resource utilization}

During the first year after ART initiation, patients utilized, on average, 9.1 months' worth of NRTI drugs and 9.0 months' worth of NNRTI drugs or protease inhibitor (PI) drugs (Table 5). Patients also utilized, on average, 5.2 months' worth of co-trimoxazole, $1.2 \mathrm{CD} 4$ tests, 1.1 blood chemistry tests, 1.2 full blood count tests, 5.4 clinic visits with a doctor or clinical officer, and 7.9 visits with a pharmacist.

As expected, patients retained in care one year after initiating ART utilized substantially more resources than those known to have died or lost to follow up, including 11.4 months' worth of NRTIs and 11.2 months' worth of NNRTIs or PIs, 6.5 months' worth of co-trimoxazole, 1.5 CD4 tests, 1.3 blood chemistry tests, 1.4 full blood count tests, 6.5 clinic visits with a doctor or clinical officer, and 9.5 visits with a pharmacist. The quantity of each resource utilized varied widely by site.

\section{Unit costs}

ARV drug costs, which were constant across sites, varied by drug regimen (Table 6). Abacavir and tenofovir were more expensive than zidovudine and stavudine, and efavirenz was more expensive than nevirapine. The cost of co-trimoxazole was modest compared to the cost of ARV drugs.

Laboratory test costs varied widely between sites. The cost per CD4 test, for example, ranged from $\$ 2.87$ at sites 1 and 2, where tests were run at a centralized, high-volume laboratory in Lusaka, to $\$ 18.16$ at site 5 where tests were run onsite at a relatively low-volume laboratory. The cost per clinic visit with each type of provider and the cost of fixed resources per month in care also varied widely between sites.

\section{Average cost per patient}

During the first year after ART initiation, the average cost per patient for the total sample was $\$ 198$ (95\% CI, \$157-\$239), ranging from \$151 (95\% CI, \$137-\$165) at site 1 to $\$ 251$ (95\% CI, \$229-\$273) at site 5 (Table 7). The average cost per patient for the subset of the sample remaining in care one year after initiating ART was \$243 (95\% CI, \$194-\$293), ranging from \$184 (95\% CI, \$172$\$ 195)$ at site 1 to $\$ 304(95 \% \mathrm{CI}, \$ 290-\$ 319)$ at site 5.

At sites 2 and 6 , where patients were followed for three years after ART initiation, the average annual cost per patient remaining in care decreased from $\$ 245$ (95\% CI, \$235-\$254) and \$296 (95\% CI, \$285-\$307) one year after initiating ART to \$233 (95\% CI, \$221-\$245) and $\$ 272$ (95\% CI, \$262-\$282) two years after initiating ART and \$221 (95\% CI, \$208-\$234) and \$270 (95\% CI, \$260-\$279) three years after initiating ART. Decreases in the cost per patient remaining in care over time are due primarily to decreases in the quantity of resources utilized over time.

Antiretroviral drugs were the largest cost component at all six sites, comprising between $38 \%$ (site 5) and $84 \%$ (site 4) of the average cost per patient for the subset of the sample remaining in care one year after initiating ART.

\section{Discussion}

The Government of Zambia revised national HIV treatment guidelines in 2007 to recommend the use of tenofovir as a standard component of first-line ART and in 2010 and 2013 to expand eligibility criteria for patients not yet on ART [3-5]. As the Government of Zambia considers new guidelines changes, with potentially large implications for program planning and budgets, information on the actual care provided under previous guidelines can help guide decision making. We used primary, patient-level data to estimate retention in care, the quantity of resources utilized, and the costs of providing ART to adults in routine clinical practice in Zambia after adoption of the 2007 national guidelines.

Patients initiated ART at a median CD4 cell count of 145 cells $/ \mu \mathrm{L} ; 59 \%$ initiated on a tenofovir-containing regimen. One year after ART initiation, $75 \%$ of patients were retained in care. Patients retained in care received, 
Table 5 Average quantity of resources utilized per patient during the first year on antiretroviral therapy

\begin{tabular}{|c|c|c|c|c|c|c|c|}
\hline Resource & Site 1 & Site 2 & Site 3 & Site 4 & Site 5 & Site 6 & All \\
\hline \multicolumn{8}{|c|}{ Quantity of resources utilized per patient for total sample } \\
\hline \multicolumn{8}{|l|}{ ARV drugs, number of patient-months } \\
\hline \multicolumn{8}{|l|}{ NRTI combinations } \\
\hline TDF 300 mg + FTC 200 mg & 3.7 & 7.6 & 7.3 & 1.3 & 4.1 & 8.8 & 5.6 \\
\hline AZT 300 mg + 3TC 150 mg & 2.6 & 0.0 & 0.8 & 0.5 & 2.2 & 1.0 & 1.1 \\
\hline ABC $300 \mathrm{mg}+3 \mathrm{TC} 150 \mathrm{mg}$ & 0.1 & 0.9 & 0.0 & 6.4 & 0.0 & 0.1 & 1.3 \\
\hline d4T $30 \mathrm{mg}+3 \mathrm{TC} 150 \mathrm{mg}$ & 2.5 & 0.6 & 0.6 & 0.5 & 1.4 & 1.1 & 1.1 \\
\hline Any NRTI combination & 8.9 & 9.1 & 8.8 & 8.7 & 7.8 & 11.1 & 9.1 \\
\hline \multicolumn{8}{|l|}{ NNRTI or PI } \\
\hline NVP 200 mg & 6.4 & 7.7 & 4.0 & 2.5 & 3.7 & 4.0 & 4.8 \\
\hline EFV 600 mg & 2.2 & 1.5 & 4.5 & 6.2 & 4.0 & 6.8 & 4.2 \\
\hline LPV/r 200/50 mg & 0.1 & 0.0 & 0.0 & 0.0 & 0.0 & 0.0 & 0.0 \\
\hline Any NNRTI or PI & 8.8 & 9.2 & 8.5 & 8.6 & 7.7 & 10.8 & 9.0 \\
\hline \multicolumn{8}{|l|}{ Non-ARV drugs, number of patient-months ${ }^{a}$} \\
\hline Co-trimoxazole 400/80 mg & 6.4 & 8.8 & 1.3 & 3.2 & 0.2 & 9.8 & 5.2 \\
\hline \multicolumn{8}{|l|}{ Laboratory tests, number of tests ${ }^{b}$} \\
\hline CD4 test & 1.5 & 1.3 & 1.4 & 0.5 & 1.5 & 1.2 & 1.2 \\
\hline Blood chemistry test $^{c}$ & 0.2 & 1.9 & 1.0 & 0.3 & 1.0 & 2.2 & 1.1 \\
\hline Full blood count $^{\mathrm{d}}$ & 1.3 & 1.2 & 1.0 & 0.4 & 2.0 & 1.3 & 1.2 \\
\hline \multicolumn{8}{|c|}{ Clinic visits with each type of provider, number of visits ${ }^{\mathrm{e}}$} \\
\hline Doctor or clinical officer & 5.0 & 5.3 & 5.3 & 3.4 & 6.6 & 7.4 & 5.4 \\
\hline Nurse & 7.4 & 6.8 & 5.4 & 4.2 & 6.6 & 7.4 & 6.3 \\
\hline Counselor & 9.2 & 9.2 & 6.1 & 7.6 & 6.6 & 6.6 & 7.6 \\
\hline Pharmacist & 9.5 & 10.0 & 6.1 & 7.2 & 6.6 & 7.3 & 7.9 \\
\hline Fixed resources, number of patient-months ${ }^{f}$ & 9.9 & 9.3 & 9.6 & 10.1 & 9.6 & 9.8 & 9.7 \\
\hline
\end{tabular}

Quantity of resources utilized per patient for subset of sample retained in care one year after initiating ART

ARV drugs, number of patient-months

NRTI combinations

TDF 300 mg + FTC 200 mg

6

9.0 5.2 1.2 .1 .2

5.4 6.3 7.6 7.9 9.7

AZT 300 mg + 3TC 150 mg

$A B C 300 \mathrm{mg}+3 T C 150 \mathrm{mg}$

$\mathrm{d} 4 \mathrm{~T} 30 \mathrm{mg}+3 \mathrm{TC} 150 \mathrm{mg}$

Any NRTI combination

$\begin{array}{lll}4.3 & 10.2 & 9.3 \\ 3.3 & 0.0 & 1.0 \\ 0.1 & 1.2 & 0.0 \\ 3.1 & 0.9 & 0.8\end{array}$

1.6

5.1

10.8

6.9

0.6

NNRTI or PI

NVP $200 \mathrm{mg}$

$\begin{array}{lllllll}7.9 & 10.3 & 5.1 & 2.9 & 4.6 & 5.1 & 6.0 \\ 2.6 & 2.0 & 5.6 & 7.4 & 5.0 & 8.1 & 5.2 \\ 0.2 & 0.0 & 0.0 & 0.0 & 0.0 & 0.0 & 0.0 \\ 10.7 & 12.3 & 10.7 & 10.2 & 9.6 & 13.2 & 11.2\end{array}$

Non-ARV drugs, number of patient-months ${ }^{a}$

Co-trimoxazole

Laboratory tests, number of tests ${ }^{\mathrm{b}}$

CD4 test

$\begin{array}{lllllll}1.8 & 1.9 & 1.6 & 0.6 & 1.7 & 1.5 & 1.5 \\ 0.1 & 2.5 & 1.0 & 0.3 & 1.0 & 2.6 & 1.3 \\ 1.6 & 1.6 & 1.0 & 0.5 & 2.2 & 1.6 & 1.4\end{array}$

Blood chemistry test ${ }^{c}$

Full blood count ${ }^{\text {d }}$ 
Table 5 Average quantity of resources utilized per patient during the first year on antiretroviral therapy (Continued)

Clinic visits with each type of provider, number of visits ${ }^{\mathrm{e}}$

Doctor or clinical officer

Nurse

Counselor

Counselor

Pharmacist

Fixed resources, number of patient-months ${ }^{f}$

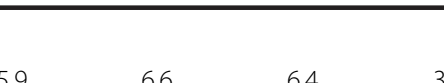

3TC: lamivudine; ABC: abacavir; ART: antiretroviral therapy; ARV: antiretroviral; AZT: zidovudine; d4T: stavudine; EFV: efavirenz; FTC: emtricitabine; LPV/r: ritonavirboosted lopinavir; NNRTI: non-nucleoside reverse transcriptase inhibitor; NRTI: nucleoside reverse transcriptase inhibitor; NVP: nevirapine; PI: protease inhibitor; TDF: tenofovir.

a Patients in our sample received other non-ARV drugs in addition to co-trimoxazole. These included, but were not limited to: multivitamins, folic acid supplements, ferrous sulfate supplements, paracetamol, fluconazole, ibuprofen, and amoxicillin.

${ }^{\mathrm{b}}$ In addition to CD4 tests, blood chemistry tests, and full blood counts, patients in our sample also received $<0.1$ on average of each of the following lab tests during the first year on ART: acid-fast bacillus, hepatitis B test, malaria test, pregnancy test, rapid plasma reagin test, and viral load test.

${ }^{\mathrm{C}} \mathrm{A}$ blood chemistry test could include any combination of the following tests: creatinine, aspartate aminotransferase, alanine aminotransferase, total bilirubin, direct bilirubin, and urea.

${ }^{d}$ A full blood count includes hemoglobin and a white blood count, among other counts.

${ }^{\mathrm{e}} \mathrm{A}$ single clinic visit for patients in our sample could include a consultation with more than one type of provider (doctor or clinical officer, nurse, counselor, or pharmacist).

fFixed resources included buildings and infrastructure, equipment, supplies, vehicles, and staff time for staff employed in the ART clinic who do not see patients.

Table 6 Unit costs (in 2011 USD) for resources utilized by adults receiving antiretroviral therapy

\begin{tabular}{|c|c|c|c|c|c|c|}
\hline Resource & Site 1 & Site 2 & Site 3 & Site 4 & Site 5 & Site 6 \\
\hline \multicolumn{7}{|c|}{ ARV drugs, cost per patient-month dispensed } \\
\hline \multicolumn{7}{|l|}{ NRTI combinations } \\
\hline TDF 300 mg + FTC 200 mg & 10.54 & 10.54 & 10.54 & 10.54 & 10.54 & 10.54 \\
\hline AZT $300 \mathrm{mg}+3$ TC $150 \mathrm{mg}$ & 8.41 & 8.41 & 8.41 & 8.41 & 8.41 & 8.41 \\
\hline $\mathrm{ABC} 300 \mathrm{mg}+3 \mathrm{TC} 150 \mathrm{mg}$ & 18.64 & 18.64 & 18.64 & 18.64 & 18.64 & 18.64 \\
\hline $\mathrm{d} 4 \mathrm{~T} 30 \mathrm{mg}+3 \mathrm{TC} 150 \mathrm{mg}$ & 3.01 & 3.01 & 3.01 & 3.01 & 3.01 & 3.01 \\
\hline \multicolumn{7}{|l|}{ NNRTI or PI } \\
\hline NVP 200 mg & 2.34 & 2.34 & 2.34 & 2.34 & 2.34 & 2.34 \\
\hline EFV $600 \mathrm{mg}$ & 4.26 & 4.26 & 4.26 & 4.26 & 4.26 & 4.26 \\
\hline LPV/r 200/50 mg & 36.70 & 36.70 & 36.70 & 36.70 & 36.70 & 36.70 \\
\hline \multicolumn{7}{|c|}{ Non-ARV drugs, cost per patient-month dispensed } \\
\hline Co-trimoxazole 400/80 mg & 0.93 & 0.93 & 0.93 & 0.93 & 0.93 & 0.93 \\
\hline \multicolumn{7}{|l|}{ Laboratory tests, cost per test ${ }^{a}$} \\
\hline CD4 test & 2.87 & 2.87 & 11.90 & 10.56 & 18.16 & 10.06 \\
\hline Blood chemistry test & 2.93 & 2.93 & 5.01 & 7.23 & 5.11 & 2.27 \\
\hline Full blood count & 2.80 & 2.80 & 2.14 & 5.24 & 5.60 & 1.11 \\
\hline \multicolumn{7}{|c|}{ Clinic visits with each type of provider, cost per visit ${ }^{b}$} \\
\hline Doctor or clinical officer visit & 1.71 & 1.82 & 0.57 & 0.37 & 2.86 & 1.01 \\
\hline Nurse visit & 0.52 & 1.01 & 0.48 & 0.40 & 1.91 & 0.59 \\
\hline Counselor visit & 0.56 & 0.15 & 0.31 & 1.00 & 2.11 & 2.21 \\
\hline Pharmacist visit & 0.42 & 0.32 & 0.29 & 0.45 & 1.50 & 0.28 \\
\hline Fixed resources, cost per month in care ${ }^{c}$ & 1.03 & 1.17 & 1.21 & 0.60 & 6.04 & 3.31 \\
\hline
\end{tabular}

3TC: lamivudine; ABC: abacavir; ARV: antiretroviral; AZT: zidovudine; d4T: stavudine; EFV: efavirenz; FTC: emtricitabine; LPV/r: ritonavir-boosted lopinavir; NNRTI: non-nucleoside reverse transcriptase inhibitor; NRTI: nucleoside reverse transcriptase inhibitor; NVP: nevirapine; PI: protease inhibitor; TDF: tenofovir; USD: United States Dollar.

aLaboratory tests for sites 1 and 2 were done at a centralized, high volume laboratory in Lusaka. Laboratory tests for sites 3, 4, 5, and 6 were done onsite.

${ }^{b}$ Clinic visit costs include the cost of staff time for staff who see patients.

${ }^{c}$ Fixed costs include the cost of buildings and infrastructure, equipment, supplies, vehicles, and staff time for staff employed in the ART clinic who do not see patients. 
Table 7 Average costs and cost breakdown for the first year on antiretroviral therapy

\begin{tabular}{|c|c|c|c|c|c|c|c|}
\hline & Site 1 & Site 2 & Site 3 & Site 4 & Site 5 & Site 6 & All patients at all sites ${ }^{\mathrm{d}}$ \\
\hline \multicolumn{8}{|l|}{ Average cost, 2011 USD (95\% Cl) } \\
\hline Average cost per patient for total sample & $151(137-165)$ & $185(168-202)$ & $166(154-179)$ & $209(192-225)$ & $251(229-273)$ & $242(224-260)$ & $198(157-239)$ \\
\hline $\begin{array}{l}\text { Average cost per patient for subset of sample } \\
\text { retained in care one year after initiating ART }\end{array}$ & $184(172-195)$ & $245(235-254)$ & $205(198-212)$ & $247(233-261)$ & $304(290-319)$ & $296(285-307)$ & $243(194-293)$ \\
\hline \multicolumn{8}{|c|}{ Breakdown of average cost per patient for subset of sample remaining in care one year after initiating ART, 2011 USD (\% of total) } \\
\hline ARV drugs ${ }^{a}$ & $123(67)$ & $166(68)$ & $148(72)$ & $207(84)$ & $116(38)$ & $178(60)$ & $159(66)$ \\
\hline Non-ARV drugs ${ }^{a}$ & $12(6)$ & $20(8)$ & $4(2)$ & $5(2)$ & $0(0)$ & $22(7)$ & $11(5)$ \\
\hline Laboratory tests & $11(6)$ & $18(7)$ & $27(13)$ & $11(5)$ & $49(16)$ & $23(8)$ & $22(9)$ \\
\hline Clinic visits ${ }^{b}$ & $26(14)$ & $27(11)$ & $11(5)$ & $17(7)$ & $67(22)$ & $33(11)$ & $28(11)$ \\
\hline Fixed resources ${ }^{c}$ & $12(6)$ & $14(6)$ & $15(7)$ & $7(3)$ & $72(24)$ & $39(13)$ & $24(9)$ \\
\hline Total & $184(100)$ & 245 (100) & 205 (100) & 247 (100) & 304 (100) & $296(100)$ & $243(100)$ \\
\hline
\end{tabular}

ART: antiretroviral therapy; ARV: antiretroviral; Cl: confidence interval; USD: United States Dollar.

Unit costs for ARV and non-ARV drugs were standardized across all sites so variation in total ARV and non-ARV drug costs between sites are due to differences in utilization.
${ }^{b}$ Clinic visits include the cost of staff time for staff who see patients.

Fixed costs include the cost of buildings and infrastructure, equipment, supplies, vehicles, and staff time for staff employed in the ART clinic who do not see patients.

${ }_{\mathrm{d}}^{\mathrm{d} e c a u s e}$ the sample size varied by site, the average cost for all patients at all sites is slightly different than the average cost for all sites. 
on average, 11.4 months' worth of ARV drugs, 1.5 CD4 tests, 1.3 blood chemistry tests, 1.4 full blood count tests, and 6.5 clinic visits with a doctor or clinical officer. The average cost for all patients over the first year after ART initiation was \$198 (95\% CI, \$157-\$239) while the average cost per patient retained in care one year after ART initiation was \$243 (95\% CI, \$194-\$293). Both the average quantity of resources utilized and unit costs varied widely by site, resulting in average costs per patient retained in care ranging from $\$ 184$ (95\% CI, \$172-\$195) at site 1 to $\$ 304$ (95\% CI, $\$ 290-\$ 319)$ at site 5 .

Our findings have several implications for program planning. First, as has been widely reported throughout Africa, Zambian patients are initiating ART late in the course of disease progression [16]. The median CD4 cell count at initiation for patients in our study, 145 cells $/ \mu \mathrm{L}$, was considerably lower than the 200 cells $/ \mu \mathrm{L}$ threshold for ART eligibility in place at the time when these patients initiated ART [3]. This suggests that many patients may have reported to care long after meeting the eligibility criteria for ART initiation. Future guidelines changes that increase the CD4 threshold for ART eligibility, and expand eligibility to a larger proportion of the HIV-infected population, may have a smaller impact than anticipated, both in terms of increased health benefits and increased program costs, if patients are not effectively identified and linked to care soon after meeting eligibility criteria.

Second, a large proportion of patients $(25 \%)$ are no longer in care within one year of initiating ART and attrition continues, at a slower rate, through three years after initiating ART. The average cost per patient in our sample for the first year on ART was $\$ 198$. If we were to estimate the average cost to produce a patient retained in care one year after initiating ART, calculated as the sum of all costs for all patients divided by just the number of patients remaining in care, it would be $\$ 265$. If all patients remained in care, we would expect an average cost per patient of $\$ 243$, greater than the average cost per patient in the study of $\$ 198$, but less than the cost to produce a patient remaining in care of $\$ 265$.

Third, actual resource utilization varied considerably from what would be expected if patients were receiving fully guideline-concordant care. At the time of this study, guidelines recommended initiation on a regimen containing tenofovir, emtricitabine, and either efavirenz or nevirapine. Only $59 \%$ of patients in our sample initiated ART on a tenofovir-containing regimen while the remaining $41 \%$ of patients initiated ART on regimens containing zidovudine, abacavir, or stavudine. Patients in our sample initiated ART between July 2007 and October 2008, soon after tenofovir became the recommended firstline ART regimen in Zambia. It is not known whether study sites were still in the process of adopting tenofovir at the time of this study or if a similar mix of regimens would be seen among patients initiating ART at the same sites today. Nevertheless, these deviations from expected, guideline-concordant utilization months after guidelines were changed are notable.

Regardless of ART regimen at initiation, patients retained in care one year after initiating ART received, on average, fewer than the 12 months' worth of ARV drugs required to ensure uninterrupted treatment. These patients also received only $1.5 \mathrm{CD} 4$ tests, 1.3 blood chemistry tests, and 1.4 full blood count tests during their first year on ART. Guidelines recommend two CD4 tests, at least two blood chemistry tests, and at least two hemoglobin and white blood cell count tests, components of a full blood count test, during the first year after ART initiation [3]. While patients utilized fewer drugs and laboratory tests than they would have under fully guideline-concordant care, they had more frequent clinical consultations than recommended by guidelines. Patients retained in care had an average of 6.5 visits with a doctor or clinical officer and 9.5 visits with a pharmacist during their first year on ART, compared to the five visits recommended in the guidelines [3]. All of these examples suggest that it may not be appropriate to assume that guidelines and practice will be identical for the purposes of program planning and budgeting.

The proportion of patients retained in care one year after ART initiation in our study, $75 \%$, is comparable to other published estimates of retention in care during the first year on ART [16]. Our estimated cost of $\$ 243$ per patient retained in care one year after ART initiation is lower than, but still comparable to, previously published estimates of the cost per patient-year of ART in Zambia. Tagar et al. estimated an average cost of $\$ 278$ per patient-year of ART (in 2010-2011) for a 30-site sample by estimating total facility-level costs at each site during the study period and dividing by an estimated number of patient-years in care at each site during the same period [17]. Bratt et al. estimated a cost per patient-year of ART ranging from $\$ 278$ to $\$ 523$ (in 2008 US dollars) by estimating unit costs from a 12-site sample and applying the unit costs to a set of resources that patients would be expected to utilize during their first year on ART under idealized clinical care conditions [9]. Marseille et al. estimated a cost of $\$ 428$ per patient-year of ART in on-site costs (in 2010 US dollars) for the average facility in their 45-site sample by applying unit costs to a mix of site- and patient-level data on resource utilization [8]. The cost per patient-year of ART was $\$ 638$ when off-site costs were included. Our study builds on these previous cost estimates by: (1) providing insight into the actual quantity of resources utilized and services received per patient at the level of the treatment site, and (2) differentiating between patients retained in care and those not retained. 
Our study has several limitations. First, results are from six sites so we cannot attribute variation in costs of care between sites to specific site-level characteristics. These sites were purposively selected to capture variation in location and size. While results from these sites provide insight into standard types of treatment sites in Zambia, they are not necessarily representative of the ART treatment program in Zambia as a whole. Second, because patients were only classified as lost to follow up if they were $\geq 3$ months late for their last scheduled consultation or medication pickup at the study endpoint, we likely underestimate true attrition during the study follow up period. Third, patient outcomes in this analysis are limited to what could be ascertained from a retrospective review of medical records. For patients no longer attending the study clinic, we could not always distinguish between those who had transferred to another clinic, died, or been lost to follow up due to incomplete records. Third, we excluded resource utilization and costs associated with inpatient care and outpatient care received prior to ART initiation. We also excluded costs incurred by the patient and costs for program management above the facility level. Cost estimates from Marseille et al., who found that only $\$ 428$ of the $\$ 638$ in total costs per patient-year of treatment were incurred on site, suggest that this exclusion of costs above the facility level may lead to a significant underestimate of the total cost of providing ART at the treatment sites in our study sample. Finally, results reflect retention in care, resource utilization, and costs for patients who initiated ART in 2007 and 2008, after the adoption of tenofovir as a standard component of firstline ART in 2007 but prior to the adoption of new HIV treatment guidelines in 2010 that increased the CD4 threshold for ART eligibility from 200 cells $/ \mu \mathrm{L}$ to 350 cells $/ \mu \mathrm{L}$ and the announcement in 2013 that Zambia would provide life-long ART to all pregnant women regardless of CD4 cell count $[4,5]$. Both changes may lead to a higher median $\mathrm{CD} 4$ cell count at initiation which could, in turn, result in changes in retention in care, resource utilization, and costs.

\section{Conclusions}

In summary, adult patients in Zambia initiate ART late in the course of disease progression and a large proportion drop out of care within one year. Unit costs for each resource and the average quantity of resources utilized vary widely by treatment site, as do the resulting average costs of care, suggesting opportunities for efficiency gains. Overall, patients utilize a different mix of resources under routine clinical conditions than if they were receiving fully guideline-concordant care. The differences between guidelines and practice highlight the importance of looking at what is actually happening, and not just what is expected to happen, to ensure effective program planning and accurate budgeting. Improving retention in care and guideline concordance, including increasing the use of tenofovir in first-line ART regimens, may lead to increases in overall treatment costs.

\section{Competing interests}

The authors declare that they have no competing interests.

\section{Authors' contributions}

$\mathrm{LL}$ and SR designed the study. CAS and KM oversaw data collection. CAS, $\mathrm{HSI}$, and $\mathrm{KM}$ analyzed the data. CAS, HSI, KM, CM, LL, BAL, and SR assisted with interpretation of results. CAS wrote the first draft of the manuscript. CAS, HSI, KM, CM, LL, BAL, and SR reviewed and edited the manuscript. All authors read and approved the final manuscript.

\section{Acknowledgements}

Although the authors remain solely responsible for the manuscript, the research benefited from the contributions of many individuals at the study sites, the Zambian Ministry of Health, the Centre for Infectious Disease Research in Zambia, the Zambia Prevention, Care and Treatment Program, and the U.S. Centers for Disease Control and Prevention. The authors thank Petan Hamazakaza, Maria Kankondo, Joshua Kasimba, Arthur Mazimba, and Philip Seidenberg for their assistance with the study. This research was funded by the President's Emergency Plan for AIDS Relief (PEPFAR) through the Centers for Disease Control and Prevention (5U2GPS001418-00).

\section{Author details}

${ }^{1}$ Center for Global Health and Development, Boston University, Crosstown Center, 3rd Floor, 801 Massachusetts Avenue, Boston, MA 02118, USA. ${ }^{2}$ Zambia Center for Applied Health Research and Development, Plot 4649 Beit Road, Rhodes Park, P.O. Box 30910, Lusaka 10101, Zambia. ${ }^{3}$ Zambian Ministry of Health, Ndeke House, P.O. Box 30205, Lusaka 10101, Zambia. ${ }^{4}$ Vanderbilt Institute of Global Health, Vanderbilt University, 2525 West End Avenue, Suite 750, Nashville, TN 37203, USA. ${ }^{5}$ Health Economics and Epidemiology Research Office, Wits Health Consortium, Department of Clinical Medicine, School of Internal Medicine, Faculty of Health Sciences, University of the Witwatersrand, Helen Joseph Hospital, Perth Road, Westdene, Johannesburg 2092, South Africa. ${ }^{6}$ Department of International Health, School of Public Health, Boston University, Crosstown Center, 3rd Floor, 801 Massachusetts Avenue, Boston, MA 02118, USA

Received: 26 July 2013 Accepted: 14 March 2014 Published: 31 March 2014

\section{References}

1. UNAIDS: Zambia HIV and AIDS estimates. [http://www.unaids.org/en/ regionscountries/countries/zambia/]

2. Government of the Republic of Zambia, Ministry of Health and the National AIDS Council: Zambia country report: Monitoring the declaration of commitment on HIV and AIDS and the universal access, Biennial report. [http://www.unaids.org/en/dataanalysis/knowyourresponse/ countryprogressreports/2012countries/ce ZM_Narrative Report.pdf]

3. Government of the Republic of Zambia, Ministry of Health: Antiretroviral therapy for chronic HIV infection in adults and adolescents: New ART protocols. [http://apps.who.int/medicinedocs/documents/s19278en/s19278en.pdf]

4. Government of the Republic of Zambia, Ministry of Health: Adult and adolescent antiretroviral therapy protocols. [http://www.who.int/hiv/pub/ guidelines/zambia_art.pdf]

5. Government of the Republic of Zambia, Ministry of Health: Lifelong antiretroviral drugs (ARV's) for all HIN positive pregnant women in Zambia: Policy guidelines for health facilities in Zambia. [http://www.emtct-iatt.org/wp-content/uploads/ 2013/02/Policy-guidelines-for-eMTCT-Option-B+_Zambia-2013-signed2.pdf]

6. Stringer JS, Zulu I, Levy J, Stringer EM, Mwango A, Chi BH, Mtonga V, Reid S, Cantrell RA, Bulterys M, Saag MS, Marlink RG, Mwinga A, Ellerbrock TV, Sinkala M: Rapid scale-up of antiretroviral therapy at primary care sites in Zambia: feasibility and early outcomes. JAMA 2006, 296(7):782-793.

7. Chi BH, Mwango A, Giganti M, Mulenga LB, Tambatamba-Chapula B, Reid SE, Bolton-Moore C, Chintu N, Mulenga PL, Stringer EM, Sheneberger, 
Mwaba P, Stringer JS: Early clinical and programmatic outcomes with tenofovir-based antiretroviral therapy in Zambia. J Acquir Immune Defic Syndr 2010, 54(1):63-70.

8. Marseille E, Giganti MJ, Mwango A, Chisembele-Taylor A, Mulenga L, Over M, Kahn JG, Stringer JS: Taking ART to scale: determinants of the cost and cost-effectiveness of antiretroviral therapy in 45 clinical sites in Zambia. PLoS One 2012, 7(12):e51993.

9. Bratt JH, Torpey K, Kabaso M, Gondwe Y: Costs of HIV/AIDS outpatient services delivered through Zambian public health facilities. Trop Med Int Health 2011, 16(1):110-118.

10. Scott CA, lyer H, Lembela Bwalya D, McCoy K, Meyer-Rath G, Moyo C, Bolton-Moore C, Larson B, Rosen S: Retention in care and outpatient costs for children receiving antiretroviral therapy in Zambia: a retrospective cohort analysis. PLoS One 2013, 8(6):e67910.

11. Weinstein MC, Siegel JE, Gold MR, Kamlet MS, Russell LB: Recommendations of the panel on cost-effectiveness in health and medicine. JAMA 1996, 276(15):1253-1258.

12. World Health Organization: Global price reporting mechanism. [http:/www.who.int/hiv/amds/gprm/en/]

13. Medical Stores Limited: Medical Stores Limited: 2010 catalogue. Lusaka, Zambia: Government of the Republic of Zambia, Ministry of Health; 2010

14. International Monetary Fund: World Economic Outlook Database. [http:// www.imf.org/external/pubs/ft/weo/2012/02/weodata/index.aspx]

15. Oanda Corporation: Historical exchange rates: daily midpoint rates; 1 January 2011 to 31 December 2011. [www.oanda.com/currency/historical-rates/]

16. Fox MP, Rosen S: Patient retention in antiretroviral therapy programs up to three years on treatment in sub-Saharan Africa, 2007-2009: systematic review. Trop Med Int Health 2010, 15(Suppl 1):1-15.

17. Tagar E, Sundaram M, Condliffe K, Over M, Assefa Y, Nyemazi JP, Matatiyo B, Chilima B, Mwanamanga R, Midiani D, Moyo C, Chitah B, Batra P, Dain M, Hurley R, Kumar R, Mayer S, McCarthy T, Shear L: The cost of scaling-up antiretroviral treatment: a costing study in 161 representative facilities in Ethiopia, Malawi, Rwanda and Zambia [abstract THPE738]. In XIX International AIDS Conference: 22-27 July 2012. Washington, DC, USA; 2012. http://pag.aids2012.org/Abstracts.aspx?AID=19714.

doi:10.1186/1471-2458-14-296

Cite this article as: Scott et al:: Retention in care, resource utilization, and costs for adults receiving antiretroviral therapy in Zambia: a retrospective cohort study. BMC Public Health 2014 14:296.

\section{Submit your next manuscript to BioMed Central and take full advantage of:}

- Convenient online submission

- Thorough peer review

- No space constraints or color figure charges

- Immediate publication on acceptance

- Inclusion in PubMed, CAS, Scopus and Google Scholar

- Research which is freely available for redistribution 